\title{
Lidil
}

Revue de linguistique et de didactique des langues

$59 \mid 2019$

Prononcer les langues : variations, émotions, médiations

\section{Acquisition des voyelles nasales du français et interférences du cantonais}

Acquisition of French Nasal Vowels and Interference of Cantonese

Junkai Li, Yi Yin et Zhihong Pu

\section{CpenEdition}

\section{Journals}

Édition électronique

URL : http://journals.openedition.org/lidil/6473

DOI : $10.4000 /$ lidil.6473

ISSN : 1960-6052

Éditeur

UGA Éditions/Université Grenoble Alpes

Édition imprimée

ISBN : 978-2-37747-090-7

ISSN : $1146-6480$

Référence électronique

Junkai Li, Yi Yin et Zhihong Pu, «Acquisition des voyelles nasales du français et interférences du cantonais », Lidil [En ligne], 59 | 2019, mis en ligne le 01 mai 2019, consulté le 10 décembre 2020. URL : http://journals.openedition.org/lidil/6473 ; DOI : https://doi.org/10.4000/lidil.6473

Ce document a été généré automatiquement le 10 décembre 2020.

(C) Lidil 


\section{Acquisition des voyelles nasales du français et interférences du cantonais $^{1}$}

Acquisition of French Nasal Vowels and Interference of Cantonese

Junkai Li, Yi Yin et Zhihong Pu

\section{Introduction}

1 La prononciation joue un rôle important pour l'intelligibilité et l'intercompréhension dans la communication orale. Depuis des décennies, les techniques de recherche fondamentale servent de volets de médiation entre les pratiques d'acquisition de la prononciation et les progrès des théories actuelles. Prenant comme exemple les travaux $\mathrm{du}$ projet «Interphonologie du français contemporain» (Detey \& Racine, 2017), la linguistique expérimentale, ainsi que la prise en compte des profils distincts des apprenants, offrent des indications précises concernant le degré d'acquisition de nouveaux phonèmes par l'apprenant en abordant les aspects articulatoire, acoustique et perceptif à l'égard de certains segments en particulier.

2 Les consonnes nasales existent dans la quasi-totalité des langues du monde entier, alors que seuls $22,6 \%$ des langues $d u$ monde possèdent des voyelles nasales dans leur inventaire phonologique ${ }^{2}$ (Maddieson \& Disner, 1984; Maddieson \& Precoda, 1990). La nasalité vocalique en français moderne représente donc un trait distinctif phonéticophonologique permettant de constituer un grand nombre de paires minimales. Les voyelles nasales du français étant beaucoup étudiées depuis des décennies (Hansen, 1998; Basset et coll., 2001 ; Amelot et coll., 2002 ; Delvaux, 2004 ; Vaissière, 2008; Garnier \& Tourmel, 2012; Vajta, 2012, etc.), la production et l'acquisition de celles-ci par les apprenants allophones dans le cadre du français langue étrangère (FLE) sont, cependant, moins touchées: Montagu (2002) a comparé l'articulation labiale des voyelles nasales postérieures du français entre les locuteurs français et américains; Detey et coll. (2010) ont travaillé avec des locuteurs japonais et espagnols sur l'identification de la voyelle 
nasale et l'évaluation du degré de "consonantisation" (présence / absence de la consonne nasale post-vocalique) de leurs réalisations; Nawafleh(2013), avec des apprenants jordaniens, constate la difficulté de perception et de production que présentent ces locuteurs en confondant les voyelles nasales entre elles et avec les voyelles orales. D'ailleurs, l'accéléromètre piézoélectrique constitue un outil potentiellement à la portée des didacticiens pour l'étude de la nasalité en FLE (Pillot-Loiseau et coll., 2011; Brkan et coll., 2012a, 2012b) ; Bustamante et coll. (2014) ont étudié la durée des voyelles nasales ainsi que le ratio des RMS (Root Mean Square) nasal et oral dans les productions d'apprenantes espagnoles et colombiennes; enfin, Nguyễn Thức (2016) a montré en quoi consistent les difficultés de l'appropriation des voyelles nasales du français par le public vietnamien.

Or, les voyelles nasales n'existent ni dans l'inventaire phonologique du mandarin ni dans celui de la plupart des langues régionales en Chine, dont le cantonais ${ }^{3}$. La nasalité n'étant pas un trait distinctif, les étudiants cantonophones ont des difficultés et les assimilent à des voyelles orales coarticulées avec des consonnes nasales $/ \mathrm{n} /, / \mathrm{m} /$ ou $/ \mathrm{y} /$ en position de coda du cantonais, selon une enquête menée auprès des professeurs et des étudiants dans des universités de la province du Guangdong (Li \& Pu, 2017).

Notre recherche consiste donc à élucider des influences du cantonais, première langue acquise (L1), sur l'apprentissage de la nasalité vocalique du français. Comment la L1 influe-t-elle sur l'acquisition de la prononciation des voyelles nasales du français? Pour répondre à cette question principale, notre recherche vise à examiner les productions orales des apprenants cantonophones de différents niveaux (élémentaire, intermédiaire et avancé) en nous appuyant sur les théories de Native Language Magnet Effect (Kuhl \& Iverson, 1995 ; Iverson et coll., 2003) et le Speech Learning Model (Flege, 1995 ; Flege et coll., 2003), par le biais des analyses en phonétique expérimentale et dans le but d'accompagner les apprenants cantonophones dans l'apprentissage de la prononciation du FLE en Chine. Cette recherche concerne trois dimensions de la prononciation: les valeurs des pics spectraux impliquant la position de la langue et des lèvres, les valeurs aérodynamiques concernant le degré de nasalité, et la durée des voyelles nasales en comparaison avec la coarticulation nasale dans la langue cantonaise.

\section{Nasalité vocalique française et coarticulation en cantonais}

5 Avant d'aborder cette recherche expérimentale, nous présentons les voyelles nasales du français et la coarticulation du cantonnais.

\subsection{Voyelles nasales du français}

6 "Un bon vin blanc », une expression récurrente dans la vie quotidienne des Français semble peu facile à prononcer pour les apprenants cantonophones de FLE, parce que l'articulation de ces quatre phonèmes nécessite un couplage entre la cavité nasale et la cavité buccale, et que les propriétés articulatoires et acoustiques de ces voyelles nasales $\mathrm{du}$ français sont particulièrement complexes, surtout parce que ce sont des monophtongues en français standard. Au niveau terminologique, il nous faut distinguer deux notions essentielles: les voyelles nasales et les voyelles nasalisées. La distinction 
entre les deux types de phonie dépend du rôle distinctif, à savoir s'il s'agit d'un phonème ou non. Une voyelle nasalisée est un allophone d'une voyelle orale dans un contexte nasal environnant qui porte sur les caractéristiques non phonémiques alors qu'une voyelle nasale est une phonie permettant de distinguer le contraste phonémique entre ces voyelles et les voyelles orales: $/[\tilde{\varepsilon} / v s / l \varepsilon /$. En français moderne, les voyelles nasales et les voyelles orales suivies d'une obstruante nasale consistent majoritairement à opposer les genres grammaticaux: /bõ/ vs/bon/, point subtil à maitriser par des apprenants allophones. La confusion de cette distinction nuirait à l'intelligibilité de la communication réussie et efficace. Enfin, à une voyelle nasale en français correspond étymologiquement une ou plusieurs voyelles orales, comme le montrent les alternances

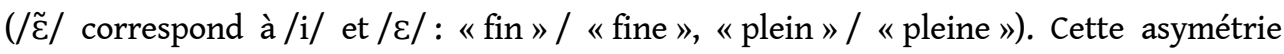
engendrerait certaines confusions cognitives au cours de l'apprentissage.

Du côté de l'articulation, un ensemble de mouvements articulatoires additionnels interviennent en complément à l'abaissement du voile du palais par rapport à la ou les orales correspondantes lors de la production des voyelles nasales du français: arrondissement des lèvres, changement d'aperture, recul de la langue dans la bouche. Pour prononcer une voyelle nasale, il faut abaisser le voile du palais jusqu'à «mi-chemin entre la langue postérieure et la paroi du pharynx, et l'air phonatoire s'écoule à la fois par les fosses nasales et la cavité buccale» (Straka, 1979, p. 503). L'abaissement du voile du palais est une condition nécessaire, mais pas suffisante à la réalisation phonétique du timbre exact des voyelles du français. Un ensemble d'articulations complémentaires y interviennent. Les propriétés acoustiques des voyelles nasales divergent donc fortement de celles de leurs correspondantes orales (Delvaux et coll., 2004). Du point de vue acoustique, Lonchamp (1979) constate que le deuxième pic spectral des nasales est régulièrement plus bas que celui des orales correspondantes: les pics spectraux correspondent à la combinaison de formants oraux et de formants / antiformants dus au couplage. Leur fréquence ne peut être directement interprétée comme une manifestation de la position de la langue. Le recul généralisé de la langue dans la cavité pharyngale pour les nasales est un phénomène bien observé en français grâce à l'imagerie médicale (Vaissière, 2008). Quant à leur production, les voyelles nasales du français sont plus longues, moins intenses, plus compactes, plus graves et plus dynamiques que les voyelles orales correspondantes. L'identification des voyelles nasales est catégorielle, liée étroitement à la connaissance phonétique qui repose sur un long apprentissage des phénomènes de covariation (Delvaux, 2004). En recourant à la terminologie de la méthode verbo-tonale d'intégration phonétique, la nasalité convoque un résonateur supplémentaire. L'air expiré s'échappe par les fosses nasales en plus de la cavité buccale.

Tableau 1. - Récapitulatif des caractéristiques phonétiques des voyelles orales et nasales du français.

\begin{tabular}{|l|l|l|}
\hline & Voyelles orales & Voyelles nasales \\
\hline Nasalité & $\begin{array}{l}\text { Voile du palais relevé } \\
\text { uniquement des formants. }\end{array}$ & $\begin{array}{l}\text { Abaissement du voile du palais. } \\
\text { Passage de l'air par les fosses nasales et la } \\
\text { cavité buccale. } \\
\text { Couplage entre les cavités orale et nasale, } \\
\text { formants et antiformants. }\end{array}$ \\
\hline
\end{tabular}




\begin{tabular}{|l|l|l|}
\hline \multirow{2}{*}{ Articulation } & $\begin{array}{l}\text { Voile du palais relevé. } \\
\text { Moins grande fermeture. } \\
\text { Plus antérieure. } \\
\text { Lèvres plus étirées. }\end{array}$ & $\begin{array}{l}\text { Abaissement de la luette. } \\
\text { Plus grande fermeture. } \\
\text { Recul de la langue dans la cavité pharyngale. } \\
\text { Lèvres moins étirées. }\end{array}$ \\
\hline Acoustique & $\begin{array}{l}\text { Second pic spectral (formant F2) } \\
\text { moins bas. } \\
\text { Moins longues, moins compactes, } \\
\text { moins graves. }\end{array}$ & $\begin{array}{l}\text { Abaissement de la hauteur de la voix. } \\
\text { Second pic spectral (formant et antiformant) } \\
\text { plus bas. } \\
\text { Plus sombres. }\end{array}$ \\
\hline
\end{tabular}

8 Selon la norme pédagogique retenue en FLE, il existe 3 voyelles nasales en français

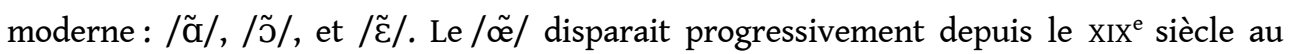
profit de la nasale $/ \tilde{\varepsilon} /$, demeurant un phonème réservé à une norme plutôt régionale (Detey \& Le Gac, 2010) ${ }^{4}$. Dans la présente étude, nous nous limitons au système des trois nasales en nous basant sur cette norme pédagogique répandue. D’ailleurs, nous prenons comme référent le « français parisien » au niveau de l'articulation des voyelles nasales, au lieu des caractéristiques des autres variations de la réalisation de ces voyelles, parce que dans le domaine du FLE, il est recommandé d'enseigner à priori un langage intelligible au plus grand nombre, c'est-à-dire de viser une prononciation la moins marquée possible pour en faire un écran à la communication le plus ténu possible (Lauret, 2007, p. 18-19).

\subsection{Coarticulation nasale en cantonais}

Comme toutes les langues ou tous dialectes chinois, qui se basent sur une écriture logogrammique, il existe une correspondance " graphie-syllabe-mot » en cantonais (nous ne tenons pas compte des tons dans la présente étude). Il en résulte que les apprenants réalisent une prononciation monosyllabique tant en L1 qu'en L2; la production et la perception de la frontière droite de syllabes jouent ainsi un rôle important dans l'apprentissage de la L2. Le cantonais partage des éléments communs avec le mandarin, langue standard et officielle chinoise, mais présente quelques caractéristiques particulières, notamment en ce qui concerne les rimes. L'analyse traditionnelle des syllabes, selon les courants linguistiques occidentaux, permet de dresser une liste des sons qui apparaissent en quatre positions dans la syllabe (voir fig. 1) :

Figure 1. - Analyse traditionnelle de la syllabe.

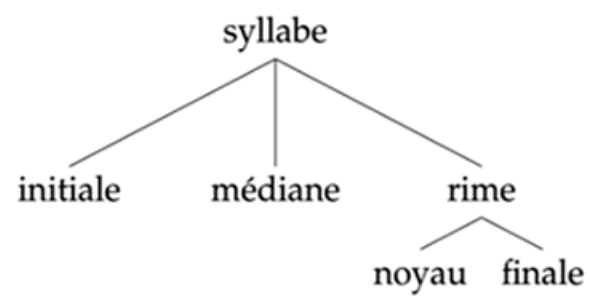

10 Cependant, dans les pratiques de l'analyse phonologique classique en Chine (yin yun xue), la syllabe des langues chinoises est divisée en deux catégories de segment principales: l'initiale (sheng $\mathrm{mu}$ ) et la finale, ou rime syllabique (yun $\mathrm{mu}$ ), cette dernière étant 
composée de la médiane (yun tou, normalement une semi-voyelle), le noyau (yun fu, normalement une voyelle) et la coda (yun wei, normalement une consonne) (voir fig. 2).

Figure 2. - Analyse syllabique du chinois (Shao, 2010).

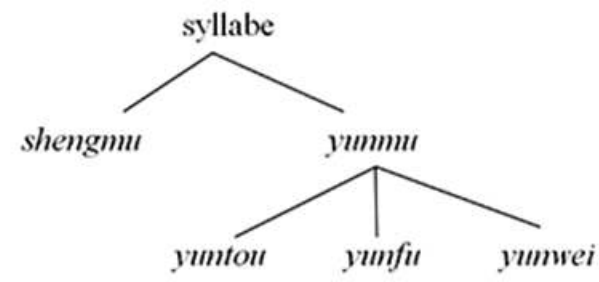

11 Contrairement à la segmentation syllabique à l'occidentale, la segmentation phonologique chinoise, dont le cantonais, est plutôt dichotomique, c'est-à-dire « initiale / rime ». La rime est considérée comme un tout insécable, ayant un noyau indispensable, une coda facultative et une médiane dont le statut reste encore polémique en cantonais (Mai, 1999; Shao, 2010). Dans les études phonologiques traditionnelles du cantonais, ainsi que dans les dictionnaires de rimes (yun shu), on classifie les sinogrammes par leurs rimes en juxtaposant les rimes simples (avec seulement le noyau) et les rimes composées (avec le noyau et la coda) dans l'inventaire vocalique (voir tableau 2).

Tableau 2. - Inventaire vocalique du cantonais.

\begin{tabular}{|c|c|c|c|c|c|c|c|c|c|c|}
\hline $\mathrm{a}$ & & $\varepsilon$ & & 0 & & $œ$ & & $\mathrm{i}$ & $\mathbf{u}$ & $\mathrm{y}$ \\
\hline ai & вi & & ei & oy & & & øу & & uy & \\
\hline $\mathrm{au}$ & $\mathrm{eu}$ & & & & ou & & & $\mathrm{iu}$ & & \\
\hline $\mathrm{am}$ & $\mathrm{em}$ & & & & & & & $\mathrm{im}$ & & \\
\hline an & en & & & on & & & øn & in & un & yn \\
\hline ay & en & $\varepsilon \eta$ & & on & on & œy & & In & & \\
\hline ap & ep & & & & & & & ip & & \\
\hline at & $\mathrm{et}$ & & & ot & & & $\varnothing \mathrm{t}$ & it & ut & $\mathrm{yt}$ \\
\hline ak & $\mathrm{ek}$ & $\varepsilon \mathrm{k}$ & & ok & ok & œk & & Ik & & \\
\hline
\end{tabular}

$\mathrm{Si}$, dans l'inventaire vocalique du cantonais, le phénomène nasal est inexistant, on observe en revanche la présence de voyelles coarticulées avec trois consonnes nasales : / $\mathrm{n} /, / \mathrm{m} /$ et $/ \mathrm{y} /$. Les voyelles coarticulées sont considérées comme des phonèmes distincts, telles que les voyelles nasales dans l'inventaire phonologique du français. Certes, il existe sans aucun doute une nasalisation des voyelles qui précèdent une consonne nasale, mais en cantonais, cette nasalité renvoie simplement à une réalisation phonétique, elle ne représente pas un trait phonémique distinctif. Iverson et coll. (2003) suggèrent que si un phonème de L2 est relativement proche d'un aimant perceptuel de la langue première, l'apprenant aura naturellement tendance à l'assimiler à la catégorie de L1. Flege (1995) et Flege et coll. (2003) affirment qu'il sera cependant de plus en plus difficile de former de nouvelles catégories correspondant aux sonorités de L2 à cause du crible phonologique (Troubetzkoy, 1969) de la L1. Inversement, il serait plus facile pour un apprenant de créer de nouvelles catégories perceptives pour des phonèmes qui ne s'apparentent pas à ceux 
de sa L1. Ainsi, les voyelles orales coarticulées avec des consonnes nasales affecteraientelles l'acquisition des voyelles nasales, étant donné la similitude de leurs valeurs tant phonétique que phonologique dans les deux langues.

À partir de cette comparaison entre les systèmes phonologiques du français et du cantonais, nous pourrions prévoir des difficultés dans l'acquisition des voyelles nasales françaises par les apprenants cantonophones. Nous tentons, ensuite, d'analyser plus précisément les indices phonétiques en ce qui concerne les interférences du cantonais au cours de l'apprentissage de la nasalité vocalique du français.

\section{Recherche expérimentale}

Nous procédons à une expérience phonétique en vue d'une réponse à notre question principale. Quatre éléments de la production orale des apprenants ont été évalués statistiquement, à savoir la durée des voyelles, les valeurs formantiques, le taux de nasalité, ainsi que la position des lèvres.

\subsection{Matériel et participants}

Dans notre étude, nous avons enregistré 18 locuteurs dont la L1 est le cantonais (un groupe de 6 personnes pour chaque niveau de l'apprentissage du français: débutant, intermédiaire et avancé ${ }^{5}$, âge moyen : 21,7 ans, $s^{2}=3,6$ ). L'ensemble de ces locuteurs étudient le français comme spécialité (option majeure) dans des universités du Guangdong, province où la population cantonophone est la plus importante de la Chine. Ils ont tous commencé à apprendre le français à partir de la première année universitaire. Les données des locuteurs ont été recueillies dans une chambre sourde, équipée d'un système d'acquisition de données aérodynamiques (PCquirer X516, Scicon), d'une caméra et d'un microphone monodirectionnel antéposé.

\subsection{Corpus}

Le corpus de prononciation avec une lecture à haute voix est composé d'une part de 3 phrases porteuses en français (lues 3 fois par les enquêtés) du type "Vous dites VCV plus que ṼCṼ deux fois [3ə vu dit VCV plys kə ṼCṼ dø fwa] », V représente /a/, / $/$ /, / / /

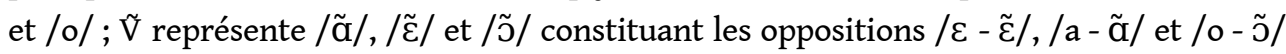
(cf. Brkan et coll., 2012b), ils se distinguent notamment par l'abaissement du voile du palais des voyelles nasales, ainsi que par une position spécifique de la langue et des lèvres. $\mathrm{C}$ représente [t], [k] et [s] qui exigent la position haute du voile du palais (Ohala, 1975) afin d'éviter de possibles vibrations des consonnes voisées sur le signal de flux nasal avant et après la voyelle. D'autre part, le corpus comprend également 3 phrases porteuses en cantonais (lues 3 fois) du type « 我同你讀 VCVC 兩次 $\left[\mathrm{yo}^{5} \mathrm{t}^{\mathrm{h}} \mathrm{Og}^{4} \mathrm{ne}^{5} \mathrm{trk}^{9} \mathrm{VCVC} l œ \mathrm{y}^{5} \mathrm{ts}^{\mathrm{h}} \mathrm{i}^{3}\right.$ ]» (Je vous lis $\mathrm{VCVC}$ deux fois), où $\mathrm{V}$ représente $/ \mathrm{a} /, / \varepsilon /$, / / / et /o/ et $\mathrm{C}$ représente /n/, / $\mathrm{m} /$ et $/ \mathrm{y} /$ (Khioe, 2003).

\subsection{Résultats et discussion}

17 Avant l'enregistrement, nous avons calibré les signaux et réglé les gains des canaux oral et nasal indépendamment afin que les niveaux des deux signaux se correspondent. Les 
données ont été segmentées et annotées manuellement à l'aide du logiciel Praat (Boersma \& Weenink, 2012).

\subsubsection{Durée des voyelles}

Nous avons mesuré respectivement la durée des voyelles orales et nasales de la production des apprenants. Par rapport aux locuteurs français natifs (Brkan et coll., 2012a), une différence significative $(p<0,05)$ entre ces deux genres de voyelles au niveau de la durée a été observée chez les trois groupes d'apprenants (voir fig. 3, 4 et 5).

Figure 3. - Durée des voyelles ( $\mathrm{ms})$ - niveau débutant $(\mathrm{N}=6)$.

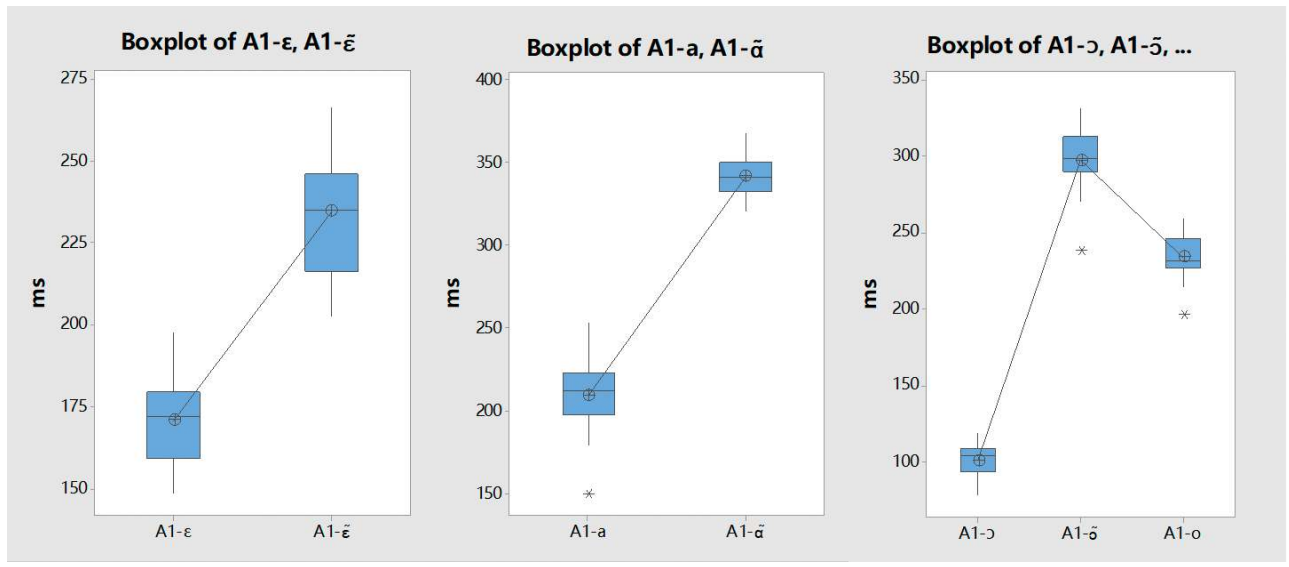

$t=-11,49 ;-20,39, f=644,84, p=0,000$

Figure 4. - Durée des voyelles (ms) - niveau intermédiaire ( $\mathrm{N}=6)$.

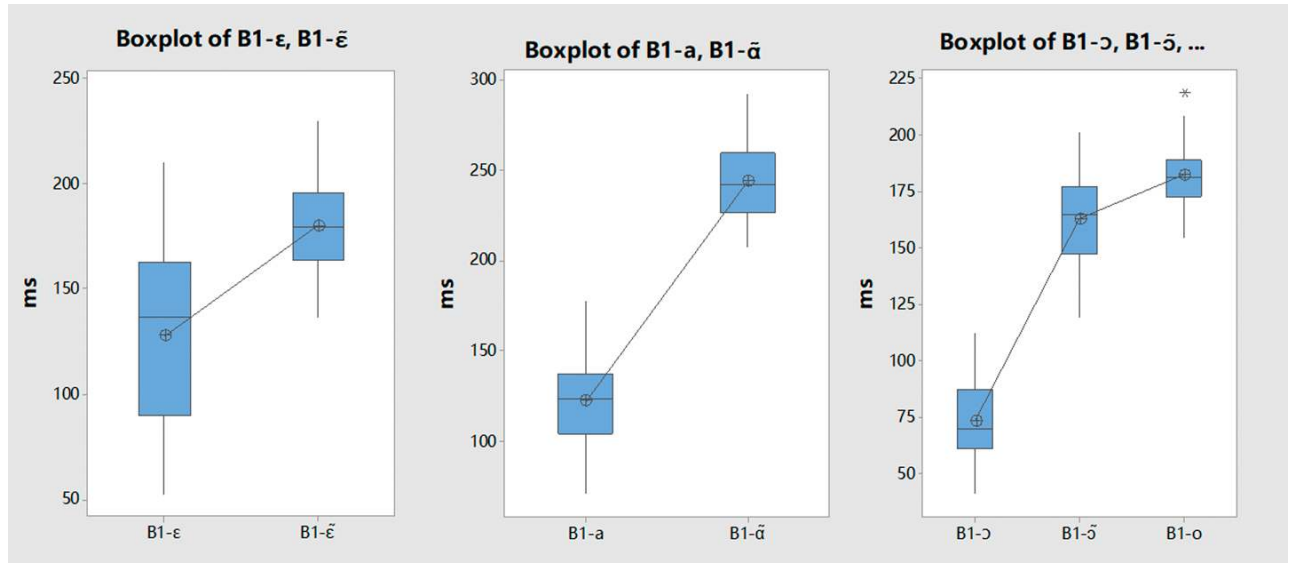

$t=-4,26 ;-14,34, f=182,92, p=0,000$ 
Figure 5. - Durée des voyelles (ms) - niveau avancé ( $\mathrm{N}=6)$.

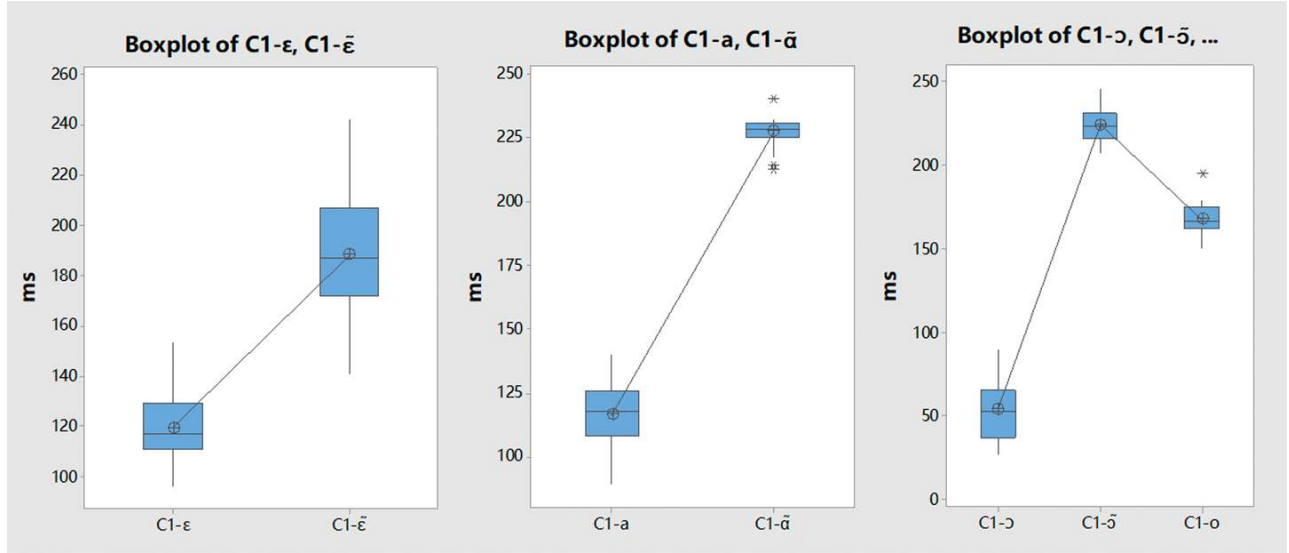

$t=-10,09 ;-30,77, f=626,62, p=0,000$

En effet, les études antérieures montrent que les francophones natifs ne prolongent pas les voyelles nasales par rapport aux voyelles sous-jacentes correspondantes en position finale (C_\#) et la durée de prononciation ne dépasse pas 200 ms en général (Pillot-Loiseau et coll., 2011 ; Brkan et coll., 2012a ; Bustamante et coll., 2014). Cependant, notre étude révèle que les apprenants, dont la $\mathrm{L} 1$ ne possède pas de voyelles nasales, tendent à prolonger la durée des voyelles pour compléter la nasalité nécessaire qui permettrait de distinguer les phonèmes oral et nasal tant en position initiale qu'en position finale. Au fur et à mesure de l'avancement de leurs études, les apprenants rapprochent peu à peu la longueur des voyelles de celles prononcées par des natifs français, mais l'écart de durée entre les voyelles nasales et orales reste net. Nous trouvons des indices d'explication dans leur langue première: la durée des voyelles nasalisées est normalement supérieure à $300 \mathrm{~ms}$ en cantonais (voir fig. 6). 


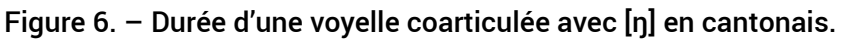

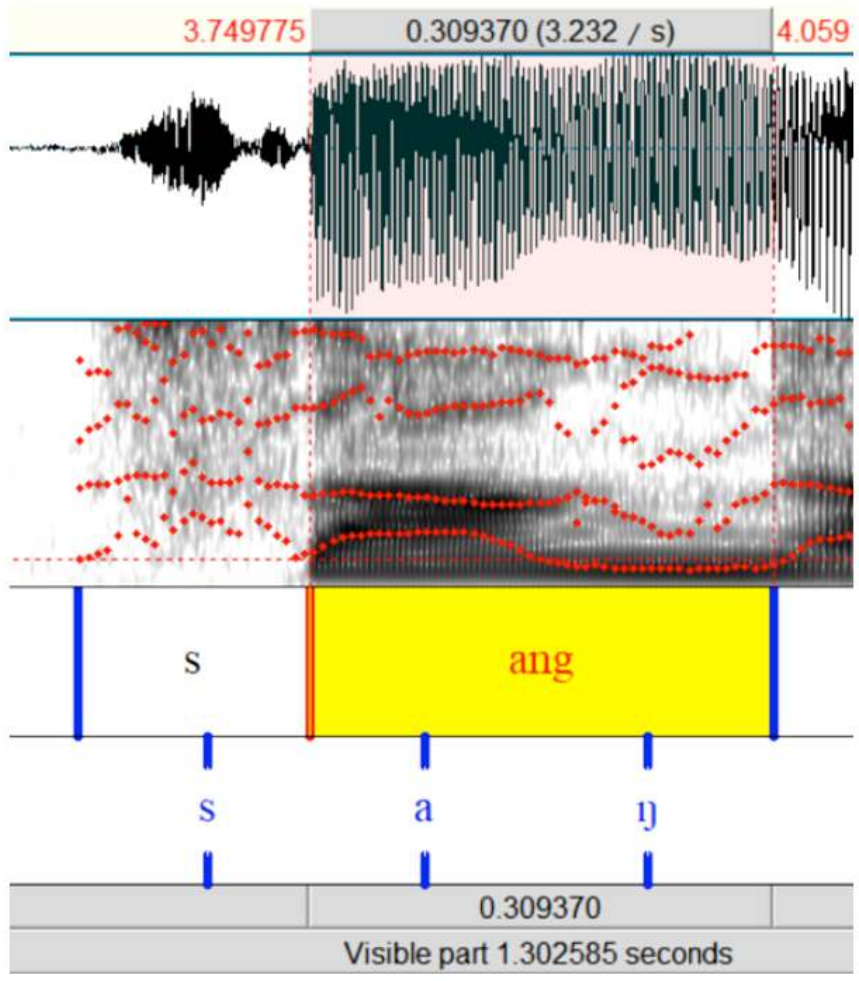

Suite à cette analyse des voyelles coarticulées avec des consonnes nasales du cantonais, nous constatons que le prolongement des voyelles nasales françaises serait dû à la longueur des voyelles coarticulées dans la langue première des apprenants. Faute de phonème nasal, l'apprenant tend à transférer la façon de prononcer "voyelle orale + consonne nasale » à la prononciation des voyelles françaises purement nasales.

\subsubsection{Valeurs formantiques}

Dans le schéma vocalique, le premier pic spectral représente la hauteur de la langue. Compte tenu du fait que le second pic spectral est le résultat de la combinaison du formant oral et des formants / antiformants venant de la cavité nasale, la valeur F2 peut être interprétée par l'auditeur comme dépendant de la position de la langue (voir fig. 7). 
Figure 7. - Schéma vocalique du natif.

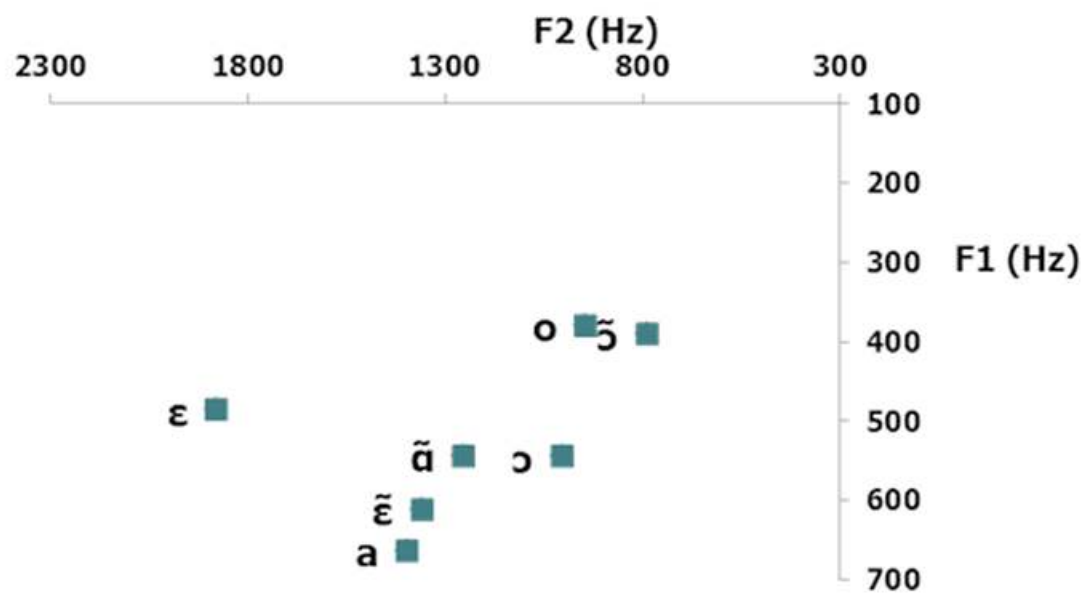

Source : Montagu (2004) cantonophones, nous remarquons que les débutants tendent à prononcer / ̃̃/ avec la langue en bas et une articulation antérieure, tandis que / $\tilde{\varepsilon} /$ se trouve au milieu de la cavité buccale ressemblant à un schwa nasalisé (voir fig. 8).

Figure 8. - Schéma vocalique des apprenants du niveau débutant.

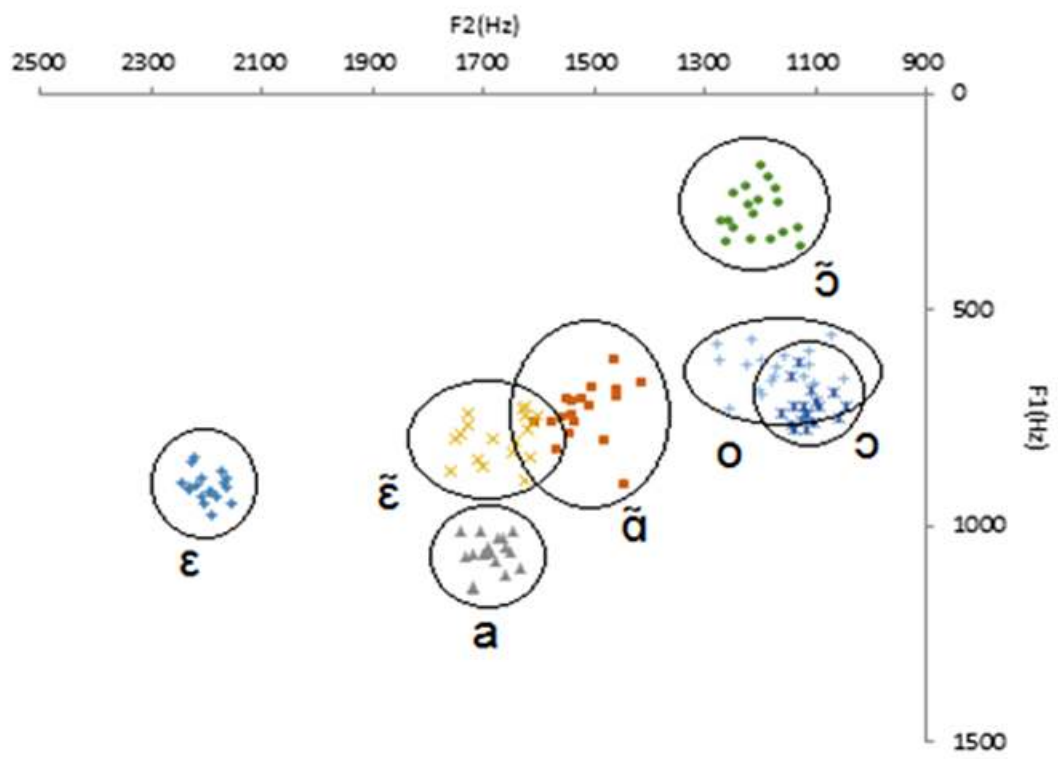

Il est intéressant d'observer, d'ailleurs, que nos apprenants cantonophones ne distinguent pas /o/ et / $/$, parce que, dans le système phonologique du cantonais, il n'existe ni le phonème de la voyelle simple /o/ ni le contraste entre /o/ et /o/. Pour les étudiants de niveau intermédiaire, la distinction entre les phonèmes se dessine. /a/ parait assez instable par rapport à d'autres sons. Les articulations de /ã/ et de /õ/ sont très proches ; $/ \tilde{\varepsilon} /$ est toujours prononcé au centre de la bouche, mais comme attendu, le pic spectral de /ã/ est plus élevé que celui de /õ/ (voir fig. 9). 
Figure 9. - Schéma vocalique des apprenants du niveau intermédiaire.

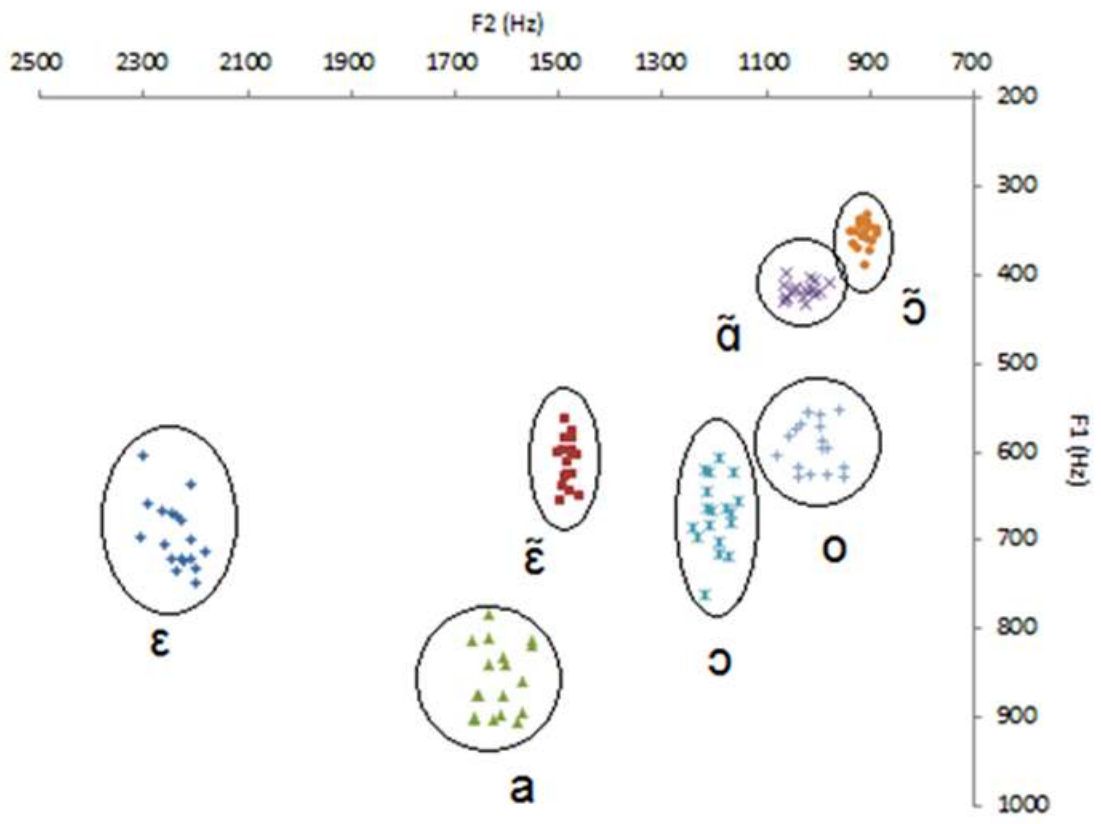

Enfin, les apprenants de niveau avancé s'approprient petit à petit la prononciation des natifs. Cependant, le point d'articulation de / ̃̃/ reste flottant et la faible distinction entre /o/ et / / persiste (voir fig. 10).

Figure 10. - Schéma vocalique des apprenants du niveau avancé.

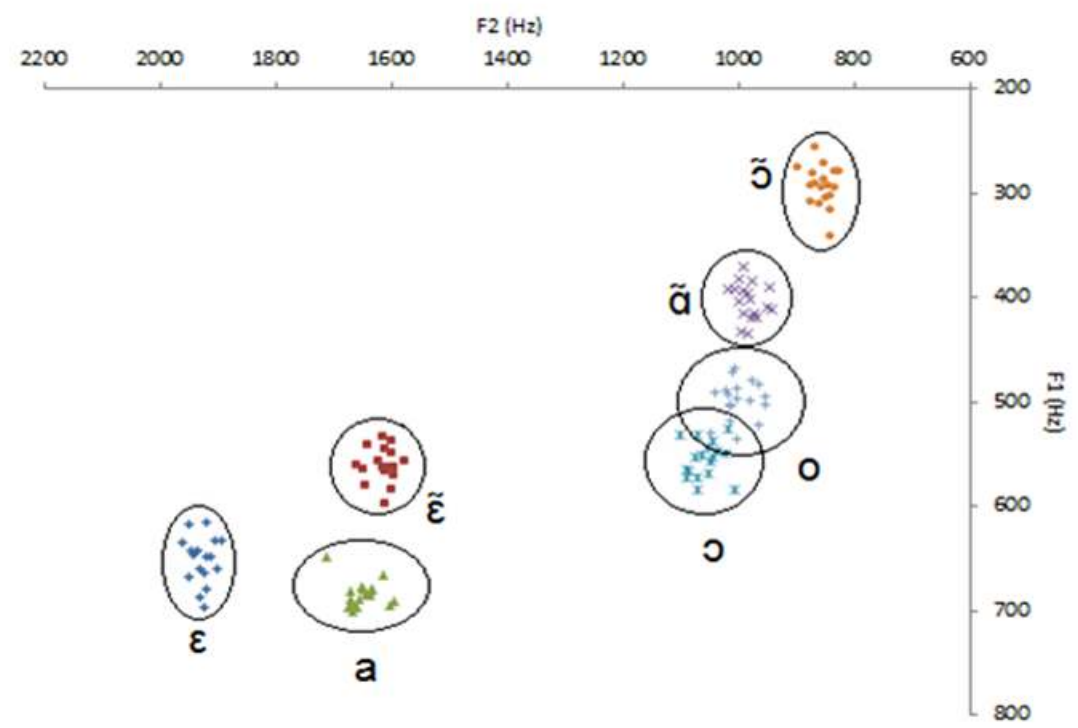

\subsubsection{Taux de nasalité}

Le taux de nasalité dans la prononciation est calculé à partir du ratio du RMS du débit d'air nasal sur le RMS total des RMS du débit d'air nasal et oral (voir fig. 11). 
Figure 11. - Calcul du taux de nasalité.

$$
T_{N}=100 \% \frac{R M S_{N}}{R M S_{N}+R M S_{O}}
$$

Les études de Brkan et coll. (2012a) montrent l'évolution de la nasalité des trois voyelles nasales en français moderne: les voyelles sont moins nasalisées en leur début. Nous remarquons, néanmoins, un taux de nasalité le plus élevé et une évolution graduelle de nasalité dès le début des voyelles chez les locuteurs français natifs (voir fig. 12).

Figure 12. - Taux de nasalité des locuteurs français natifs.

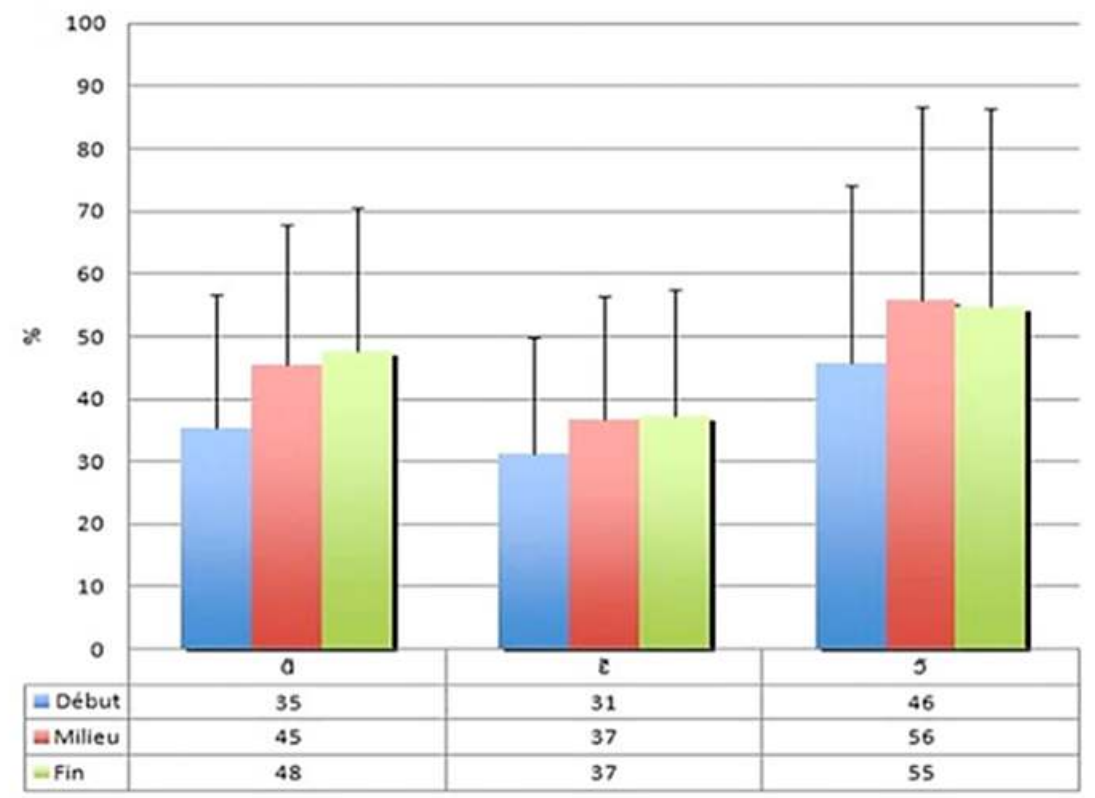

Source : Brkan et coll. (2012a)

Cependant, suite à une analyse aérodynamique de la nasalité des voyelles nasales prononcées par nos apprenants cantonophones, nous observons un taux de nasalité inférieur par rapport aux locuteurs natifs (voir fig. 13). 
Figure 13. - Nasalité des apprenants cantonophones.

\section{Evolution de la nasalité des apprenants}

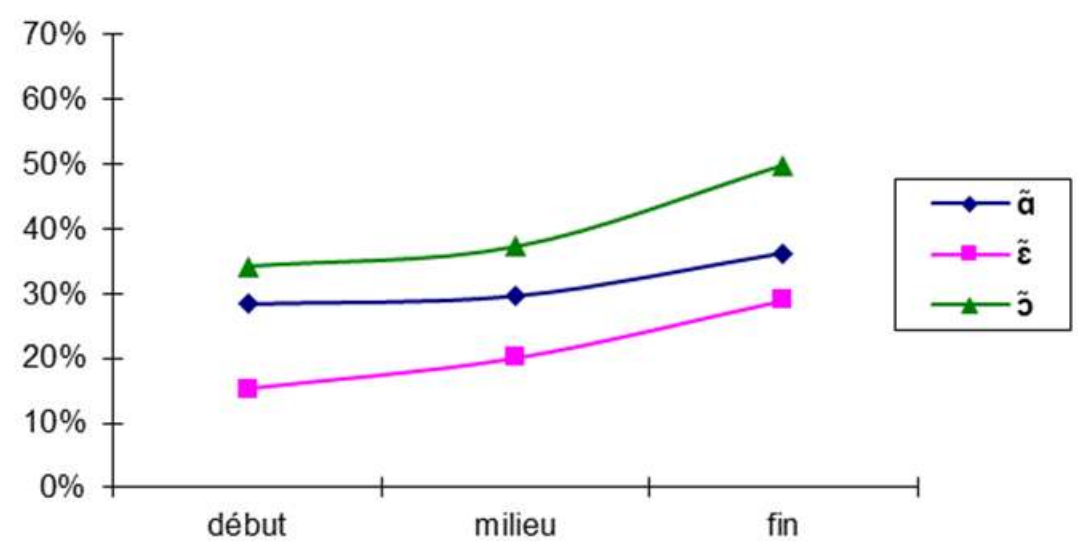

D'ailleurs, il est à noter que les locuteurs français natifs tendent à nasaliser la voyelle nasale peu de temps après le début de la voyelle, et le taux de nasalisation atteint son sommet très rapidement même au milieu de la prononciation (Amelot, 2004; Brkan et coll., 2012a). Inversement, nos apprenants n'arrivent pas à nasaliser la voyelle d'une façon efficace et le taux de nasalisation n'atteint son sommet qu'à la fin du segment, ce qui relève bien de l'épenthèse d'une obstruante nasale (voir fig. 14). Ce phénomène correspond aux caractéristiques phonétiques des voyelles coarticulées du cantonais.

Figure 14. - Débit d'air nasal d'une apprenante cantonophone.

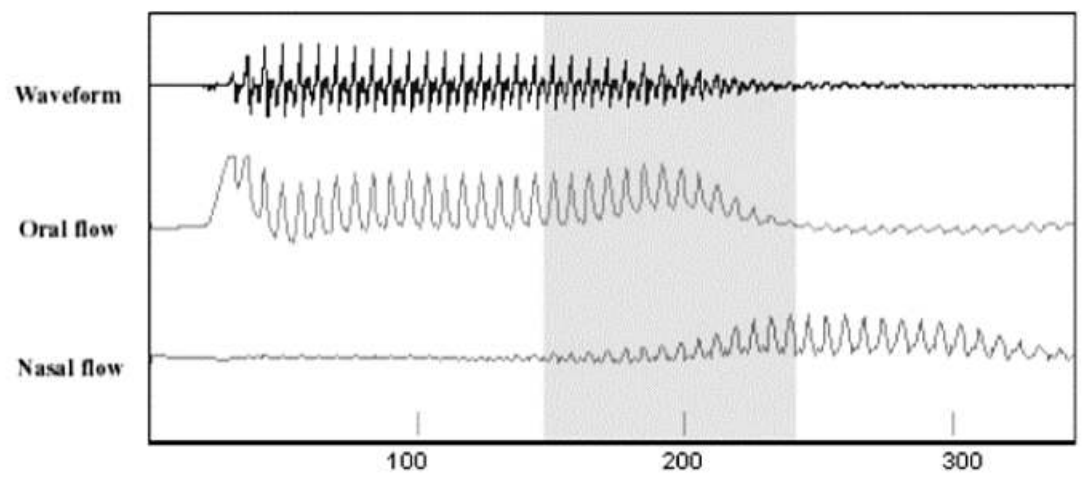

À travers cette expérimentation, nous pourrions croire que le cantonais, en tant que L1 des apprenants cantonophones, exerce phonologiquement une influence considérable en ce qui concerne la nasalité sur leur apprentissage de la prononciation du français. Comme les caractéristiques articulatoires de la voyelle nasale et la voyelle coarticulée se ressemblent beaucoup, cette dernière se transfère facilement dans l'appropriation des voyelles nasales du français.

\subsubsection{Position des lèvres}

En ce qui concerne la position des lèvres et du point d'articulation des voyelles nasales, il a été observé chez les locuteurs natifs l'ouverture des lèvres et l'abaissement de la langue 
pour le son / ̃̃/; l'aperture des lèvres et le soulèvement de la langue pour / $\tilde{\varepsilon} /$; le surarrondissement et la protrusion des lèvres et le recul important de la langue en haut et en arrière pour / ̃̃/ (Delvaux, 2004) (voir fig. 15).

Figure 15. - Position des lèvres et point d'articulation des natifs.
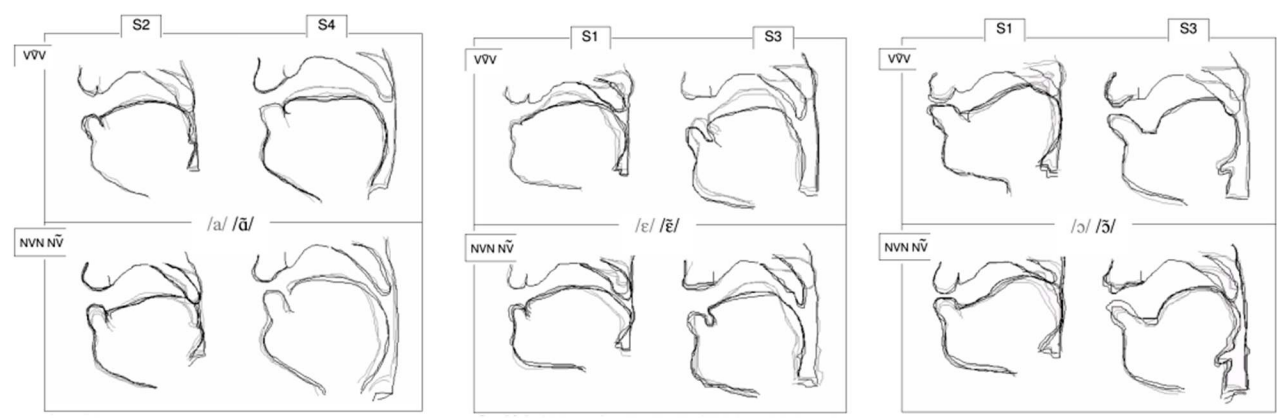

Source : Delvaux (2004)

Cependant, par rapport aux locuteurs natifs, nous observons chez nos apprenants les phénomènes suivants : les lèvres sont moins ouvertes et moins arrondies pour le son /ã / ; la langue demeure en contact avec les dents supérieures pour $/ \tilde{\varepsilon} /$; la bouche reste ouverte et les lèvres beaucoup moins arrondies pour / ̃̃/ (voir fig. 16).

Figure 16. - Position des lèvres et point d'articulation de l'apprenante.

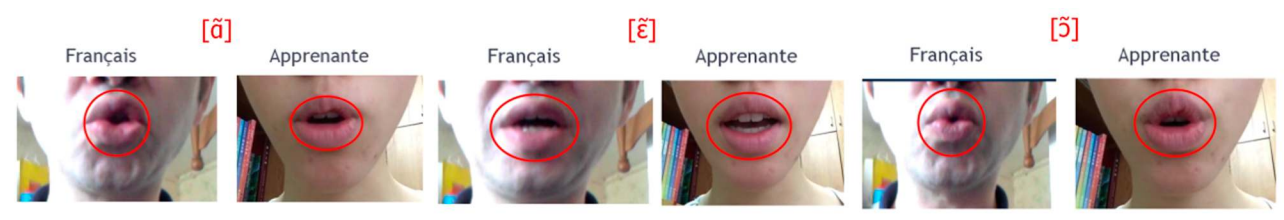

En bref, dans le but de clarifier toute l'influence du cantonais sur l'apprentissage des voyelles nasales du français, nous avons établi un tableau comparatif et récapitulatif (voir tableau 3).

Tableau 3. - Récapitulatif des incidences du cantonais sur l'apprentissage des voyelles nasales du français.

\begin{tabular}{|c|c|c|}
\hline & français des natifs & $\begin{array}{l}\text { interlangue des apprenants } \\
\text { cantonophones }\end{array}$ \\
\hline $\begin{array}{l}\text { niveau } \\
\text { phonologique }\end{array}$ & $\begin{array}{l}\text { Voyelle nasale et orale en opposition } \\
\text { phonologique; voyelle nasale et } \\
\text { "voyelle }+ \text { consonne nasale" en } \\
\text { opposition phonologique. }\end{array}$ & $\begin{array}{l}\text { Voyelle nasale inexistante; } \\
\text { voyelle nasale et consonne nasale } \\
\text { en coda en opposition } \\
\text { phonologique. }\end{array}$ \\
\hline $\begin{array}{l}\text { niveau } \\
\text { phonétique }\end{array}$ & $\begin{array}{l}\text { Durée des voyelles orales et nasales } \\
\text { distincte en position initiale et en position } \\
\text { inter-consonantique mais pas de } \\
\text { différence significative en position finale. }\end{array}$ & $\begin{array}{l}\text { Durée des voyelles nasales plus } \\
\text { longue. } \\
\text { Épenthèse de l'obstruante nasale } \\
\text { à la fin de la syllabe. }\end{array}$ \\
\hline
\end{tabular}




\begin{tabular}{|c|c|}
\hline $\begin{array}{l}\text { /ã/ : ouverture des lèvres et abaissement } \\
\text { de la langue. }\end{array}$ & $\begin{array}{l}\text { /ã / ouverture des lèvres moins } \\
\text { importante et position de la } \\
\text { langue instable. }\end{array}$ \\
\hline $\begin{array}{l}/ \tilde{\varepsilon} /: \text { aperture des lèvres et soulèvement } \\
\text { de la langue. }\end{array}$ & $\begin{array}{l}/ \tilde{\varepsilon} / \text { : langue en contact avec les } \\
\text { dents supérieures; lèvres non } \\
\text { arrondies; position de la langue } \\
\text { plus basse. }\end{array}$ \\
\hline $\begin{array}{l}/ \tilde{D} / \text { : sur-arrondissement et protrusion des } \\
\text { lèvres et recul important de la langue en } \\
\text { haut et en arrière. }\end{array}$ & $\begin{array}{l}/ \tilde{o} /: \text { bouche trop ouverte et } \\
\text { lèvres beaucoup moins arrondies. }\end{array}$ \\
\hline
\end{tabular}

\section{Conclusion et perspectives} tant que L1 exerce des interférences sur l'apprentissage de la prononciation des voyelles nasales du français. Les apprenants éprouvent des difficultés à construire la catégorie des voyelles nasales : la position de la langue et des lèvres diffèrent de celle du natif pour les trois phonèmes, la durée de leur prononciation se distingue de leur voyelle orale correspondante en certains contextes et le taux de nasalité reste insuffisant par rapport au français natif. Les résultats de notre analyse expérimentale justifient les hypothèses des modèles Native Language Magnet Effect et le Speech Learning Mode: il est difficile d'établir une catégorie phonémique en L2 similaire à celle de la L1. Les caractéristiques découvertes de l'interlangue des apprenants cantonophones du français expliquent les difficultés de ces derniers dans la prononciation des voyelles nasales. Par conséquent, il semblerait utile de prendre en compte ces caractéristiques dans l'enseignement du français aux cantonophones afin d'optimiser davantage leur apprentissage.

Toutefois, le faible nombre des données que nous avons recueillies présente une limitation de notre travail et le choix des stimuli dans différents contextes phonologiques reste à préciser pour de futures recherches. Par ailleurs, il serait judicieux de prendre en considération d'autres tâches de production : répétition et parole spontanées, etc. Enfin, un futur travail consisterait à intégrer la perception de ces voyelles nasales afin d'étudier la variabilité observée dans la production.

En guise de conclusion, soulignons à nouveau qu'il est essentiel à nos yeux de tenir compte de l'interférence de la L1 des apprenants dans l'enseignement-apprentissage de la prononciation. À ce titre, cette étude constituerait un apport dans la recherche sur la production segmentale du FLE en Chine, et les données ici présentées pourraient permettre des comparaisons avec d'autres apprenants sinophones duFLE et des recherches plus approfondies sur l'acquisition des autres segments. 


\section{BIBLIOGRAPHIE}

Amelot, Angélique, Basset, Patricia, Crevier-Buchman, Lise \& Roubeau, Bernard. (2002, juin). Nasalité en français spontané : mesures aérodynamiques et fibroscopiques, études préliminaires. Communication présentée aux «XXIV es Journées d'études sur la parole », Nancy, France.

Amelot, Angélique. (2004). Étude aérodynamique, fibroscopique, acoustique et perceptive des voyelles nasales $d u$ français (Thèse de doctorat). Université Sorbonne nouvelle Paris 3, Paris.

BASSET, Patricia, Amelot, Angélique, Vaissière, Jacqueline \& RoubeAu, Bernard. (2001). Nasal Airflow in French Spontaneous Speech. Journal of the International Phonetic Association, 31(1), 87-99. BLOT, Pauline \& KHONSARI, Roman Hossein. (2009). Mesure du flux nasal et aérophonoscopie. Revue de stomatologie et de chirurgie maxillo-faciale, 110(2), 89-93.

Boersma, Paul \& WeeninK, David. (2012). Praat: Doing Phonetics by Computer (Version 5.3. 15). Disponible en ligne sur le site de l'University of Amsterdam, Phonetics Sciences : < www.fon.hum.uva.nl/praat>.

BRKAN, Altijana, Amelot, Angélique \& Pillot-LoISEAU, Claire. (2012a). Utilisation d'un accéléromètre piezoélectrique pour l'étude de la nasalité du français langue étrangère. Actes de la Conférence conjointe JEP-TALN-RECITAL 2012, 1, 689-696.

BRKAN, Altijana, PILLOT-LoISEAU, Claire \& AMELOT, Angélique. (2012b). Étude de la nasalité du FLE à l'aide d'un accéléromètre piézoélectrique chez les locutrices natives et bosniaques : comparaison des données du corpus IPFC et d'un corpus spécifique. Communication présentée lors des « Journées IPFC (Interphonologie du français contemporain) », Paris, France.

Bustamante, David Alejandro, Amelot, Angélique \& Pillot-Loiseau, Claire. (2014). Étude de la production des voyelles nasales du français chez des apprenantes espagnoles et colombiennes. Actes des XXX ${ }^{e s}$ Journées d'études sur la parole (JEP), 576-580.

DeLATTRE, Pierre. (1959). Rapports entre la durée vocalique, le timbre et la structure syllabique en français. French Review, 32(6), 547-552.

Delvaux, Véronique. (2004). Contrôle et connaissance phonétique : les voyelles nasals du français (Thèse de doctorat, Université libre de Bruxelles, Bruxelles). Disponible en ligne sur < www.researchgate.net/publication/266183466[...]> (consulté le 29 mai 2018).

DelvauX, Véronique, Demolin, Didier, Soquet, Alin \& KingSTon, John. (2004). La perception des voyelles nasales du français. Actes des XXV es JEP, 157-160.

Delvaux, Véronique, Demolin, Didier, Harmegnies, Bernard \& Soquet, Alain. (2008). The Aerodynamics of Nasalization in French. Journal of Phonetics, 36(4), 578-606.

DETEY, Sylvain \& LE GAC, David. (2010). Le français de référence : quels locuteurs. Dans S. Detey, J. Durand, B. Laks \& C. Lyche (dir.), Les variétés du français parlé dans l'espace francophone : ressources pour l'enseignement (p. 167-180). Paris : Ophrys.

Detey, Sylvain, Racine, Isabelle, KAWAGuchi, Yuji, ZAY, Françoise \& BuEHLER, Nathalie. (2010). Évaluation des voyelles nasales en français L2 en production : de la nécessité d'un corpus multitâches. Actes du $2^{e}$ Congrès mondial de linguistique française, 1289-1301. 
DETEY, Sylvain \& RACINE, Isabelle. (2017). Towards a Perceptually Assessed Corpus of Non-Native French: The Interphonology of Contemporary French (IPFC) Project Illustrated with a Longitudinal Study of Japanese Learners' $[\mathrm{b}-\mathrm{v}]$ Productions. International Journal of Learner Corpus Research, 3(2), 223-249.

FLEGE, James Emil. (1995). Second Language Speech Learning: Theory, Findings, and Problems. Dans W. Strange (dir.), Speech Perception and Linguistic Experience: Issues in Cross-Language Research (p. 233-277). Baltimore, MD : York Press.

FLEGE, James Emil, SCHIRRU, Carlo \& MAcKAy, Ian R. A. (2003). Interaction between the Native and Second Language Phonetic Subsystems. Speech communication, 40(4), 467-491.

FUnG, Wing-Nga \& LAU, Sze-Lok. (2001, septembre). A Physiological Analysis of Nasals and Nasalization in Chinese. Communication présentée au colloque « EUROSPEECH-2001 », Aalborg, Danemark.

GARNIER, Emmanuelle \& TOURMEL, Manon. (2012). Établissement de normes de nasalance sur une population adulte de langue maternelle française ne présentant pas de rhinolalie (Mémoire de master, Université de Genève, Genève). Disponible en ligne sur <https://archive-ouverte.unige.ch/ unige:23489> (consulté le 29 mai 2018).

Guo, Lei. (2011). Étude de la nasalité du chinois basée sur l'analyse par nasomètre (Thèse de doctorat). Université des minorités du Nord-Ouest, Lanzhou.

HANSEN, Anita Berit. (1998). Les voyelles nasales du français parisien moderne : aspects linguistiques, sociolinguistiques et perceptuels des changements en cours. Copenhague : Museum Tusculanum Press. HANSEN, Anita Berit. (2001). Les changements actuels des voyelles nasales du français parisien : confusions ou changement en chaîne ? La linguistique, 37(2), 33-48.

HUANG Qiuhua et coll. (2016). Analyse des consonnes nasales du cantonais à l'aide du nasomètre. Revue de l'Université des minorités du Nord-Ouest, 2, 37-42.

IVERSON, Paul et coll. (2003). A Perceptual Interference Account of Acquisition Difficulties for Non-Native Phonemes. Cognition, 87(1), B47-B57.

KHIOE, Fung-Wah Beatrice. (2003). Temporal Properties of the Nasals and Nasalization in Cantonese. Dans Proceedings of Eurospeech 2003 (p. 2373-2376). Genève : ISCA.

KuHL, Patricia Katherine \& IVERSON, Paul. (1995). Linguistic Experience and the "Perceptual Magnet Effect". Dans W. Strange (dir.), Speech Perception and Linguistic Experience: Issues in CrossLanguage Research (p. 121-154). Baltimore, MD : York Press.

LI, Junkai \& Pu, Zhihong. (2017). Méthode syllabique dans l'enseignement de la prononciation du français en Chine. Communication présentée au « $6^{\mathrm{e}}$ Colloque international de la didactique des langues secondes », Montréal, Canada.

LAURET, Bertrand. (2007). Enseigner la prononciation du français : questions et outils. Paris : Hachette.

LONCHAMP, François. (1979). Analyse acoustique des voyelles nasales françaises. Verbum : revue de linguistique publiée par l'université Nancy 2, II, 9-54.

MADDIESON, Ian \& DiSNER, Sandra. (1984). Patterns of Sounds. Cambridge : Cambridge University Press.

MAdDIESON, Ian \& PreCodA, Kristin. (1990). Upsid-pc. The UCLA Phonological Segment Inventory Database.

MAI, Yun. (1999). Questions on the Glides in Cantonese. Journal of Sun Yat-sen University, 4, 66-71. 
MonTAGU, Julie. (2002). L'articulation labiale des voyelles nasales postérieures du français : comparaison entre locuteurs français et anglo-américains. Actes des XXIV ${ }^{e s} J E P, ~ 253-256$.

MonTAGU, Julie. (2004). Les sons sous-jacents aux voyelles nasales en français parisien : indices perceptifs des changements. Actes des Journées d'études sur la parole (JEP), 4, 385-388.

NAWAFLEH, Ahmad. (2013). Difficultés de prononciation et de perception de voyelles du français : apprenants arabophones. Paris : Presses académiques francophones.

NGUYỄN THức, Thanh Tin. (2016). Difficulté d'appropriation des voyelles nasales du français. Tạp chí Khoa học, 4(82), 41-48.

OHALA, John J. (1975). Phonetic Explanations for Nasal Sound Patterns. Dans C. A. Ferguson, L. M. Hyman \& J. J. Ohala (dir.), Nasalfest: Papers from a Symposium on Nasals and Nasalization (p. 289-316). Stanford : Stanford University Language.

Pillot-Loiseau, Claire, Amelot, Angélique \& Fredet, Florentina. (2010). Apports de la phonétique expérimentale à la didactique de la prononciation du français langue étrangère. Cahiers de l'APLIUT, XXIX(2), 75-88.

Pillot-Loiseau, Claire, AMELOT, Angélique \& BRKAN, Altijana. (2011). Utilisation d'un accéléromètre piézoélectrique pour l'étude de la nasalité du FLE. Communication présentée à la « Journée IPFC », Paris, France.

SHAO, Huijun. (2010). On the Glides $i$ and $u$ in Cantonese. Journal of Jinan University, 6, 116-122.

STRAKA, Georges. (1979). Les sons et les mots : choix d'études de phonétique et de linguistique. Paris : Klincksieck.

TRoubetZKoy, Nikolaï. (1949). Principes de phonologie (J. Cantineau, trad., 1986). Paris : Klincksieck.

VAISSIÈRE, Jacqueline. (1995, juillet). Nasalité et phonétique. Communication présentée au

«Colloque sur le voile pathologique », Lyon, France.

VAISSIÈRE, Jacqueline. (2008). X Aspects aérodynamiques et acoustiques de la nasalité. Communication présentée à l'« École d'été Porquerolles », Porquerolles, France.

VAJTA, Katharina. (2012). Autant en emporte le vin, ou : de l'importance des voyelles nasales. Moderna språk, 106(1), 145-156.

WALTER, Henriette. (1994). Variétés actuelles des voyelles nasales du français. Communication \& cognition. Monographies, 27(1-2), 223-235.

\section{NOTES}

1. This work is supported by Guangdongsheng jiaoyuting "Yuanrenzhi celue yu zizhu xuexi yanjiu" (Yue jiaogaohan [2018] 180 hao B-2018-1511) and 2018 Guangzhoushi zhexue shehuikexue keti "Wangluo ship in shijiao xia de Guangzhou xingxiang 'zisu' yu 'tasu' yanjiu" (2018GZQN59).

2. Cf. UCLA Phonological Segment Inventory Database. Disponible en ligne sur <http:// web.phonetik.uni-frankfurt.de/upsid_find.html>.

3. Le cantonais, ou langue Yue, est un dialecte chinois influent qui l'emporte sur le mandarin chez les autochtones dans les provinces de Guangdong et de Guangxi dans la Chine du Sud. Il est non seulement l'une des langues officielles de Hong Kong et de Macao, mais aussi une langue véhiculaire chez des communautés de Chinois d'outre-mer avec plus de 70 millions de locuteurs 
selon Ethnologue (16 éd., B. F. Grimes Editor, Summer Institute of Linguistics Inc., 2009, cf. < www.axl.cefan.ulaval.ca/Langues/1div_inegal_tablo1.htm>).

4. En Chine, la plupart des enseignants tendent à maintenir la distinction entre / $\tilde{\varepsilon} /$ et / $\tilde{e} /$ dans leurs pratiques pédagogiques.

5. En Chine, le $1^{\text {er }}$ cycle des études universitaires aboutissant à une licence en langue française compte 4 ans (8 semestres de 16 semaines), avec le test national dit «niveau 4 » (approximativement B1-B2) à la fin de la $2^{\mathrm{e}}$ année et le test «niveau 8 » (approximativement B2C1) à la fin de la $4^{\mathrm{e}}$ année. Le $2^{\mathrm{e}}$ cycle, qui se termine par l'obtention d'un master, dure 2 ou 3 ans selon les universités. Nous définissons les niveaux de nos sujets comme suit: niveau débutant: étudiants de $1^{\text {re }}$ année (A1-A2); niveau intermédiaire : étudiants de $3^{\mathrm{e}}$ année qui ont passé le test « niveau 4 » (B1-B2); niveau avancé : diplômés du $1^{\mathrm{er}}$ cycle ou étudiants du master (B2-C1).

\section{RÉSUMÉS}

Les voyelles nasalisées par coarticulation sont communément trouvées dans de nombreuses langues ; cependant, les voyelles nasales en tant que phonèmes indépendants n'existent que dans peu de langues. Avec 3 voyelles nasales et une position spécifique de la langue et des lèvres, les voyelles nasales $\mathrm{du}$ français constituent une difficulté importante pour les apprenants cantonophones. Cet article vise à examiner les caractéristiques de l'interlangue des voyelles nasales françaises produites par des apprenants cantonophones. En comparant les taux de nasalité, la longueur des voyelles, les pics spectraux (formants) et la position des lèvres entre les apprenants cantonophones et les locuteurs natifs du français, nous avons remarqué des influences de la coarticulation nasale en L1 (le cantonais) sur l'interlangue des voyelles nasales françaises. Les résultats de l'analyse sont les suivants : 1) il existe une différence significative au niveau du taux de nasalité entre les apprenants et les locuteurs natifs enquêtés ; 2) la L1 joue un rôle important sur la longueur des voyelles et les valeurs maximales de la nasalité de la production des voyelles nasales; 3) les apprenants éprouvent des difficultés dans l'arrondissement au cours de la production de certaines voyelles nasales françaises.

The coarticulatory nasalized vowels can be commonly found in many languages in the world. Meanwhile, regarded as independent phoneme, the nasal vowels are present only in few languages. Featured by an important rate of nasalance and the specific position of the tongue and the lips, the French nasal vowels bring about some difficulties for Chinese students. This article aims at examining the characteristics of the interlanguage of French nasal vowels produced by Cantonese learners studying French in China. Experimental phonetics methodologies are used in this research in order to compare the nasalance, the duration of vowels, the formants and the position of the lips between the Cantonophone learners and the native French speakers. The influence of the nasal coarticulation in the learner's L1 on the interlanguage of the nasal vowels in French is observed. The results of analysis show that: 1) there exists a significant difference in terms of nasalance in nasal vowels between the learners and the native French speakers; 2) L1 plays an important role in the duration of vowels and the maximum values of nasalance of French nasal vowels; 3 ) the learners experience difficulties in the roundedness of certain vowels. 
INDEX

Keywords : nasal vowel, nasalized vowel, coarticulation, French, Cantonese

Mots-clés : voyelle nasale, voyelle nasalisée, coarticulation, français, cantonais

\section{AUTEURS}

JUNKAI LI

Université Sun Yat-sen, Chine

YI YIN

Université Sorbonne Nouvelle Paris 3, France

ZHIHONG PU

Université Sun Yat-sen, Chine

puzhihong@hotmail.com 